\title{
Alcoolismo feminino: um estudo de suas peculiaridades Resultados preliminares
}

\author{
Alcoholism in women: a study of its peculiarities. Preliminary results
}

Beatriz Aceti Lenz Cesar

\section{Resumo}

Objetivo: Apresentar os resultados parciais de um estudo qualitativo realizado com mulheres alcoolistas, discutir as peculiaridades do alcoolismo feminino e a importância do seu significado nos serviços especializados. Métodos: Foram realizadas entrevistas semi-estruturadas com nove mulheres que se tratavam no serviço de alcoolismo de uma instituição pública. Resultados: Observou-se comportamento particular de beber, violência doméstica e maior adesão ao tratamento quando eram participantes de um grupo composto somente por mulheres. Conclusão: 0 estudo apontou a relevância de tentar compreender 0 alcoolismo feminino com todas as suas peculiaridades e a possivel relação dessa compreensão com os tratamentos oferecidos.

Palavras-chave: mulher, alcoolismo, gênero, violência, beber feminino.

\begin{abstract}
Objective: To present preliminary results of a qualitative study carried out with women with alcohol use disorders, to assess the peculiarities of alcoholism in women, and to point out its meanings in specialized services. Methods: Semi-structured interviews were carried out with nine women at an alcohol abuse disorders clinic in a public psychiatric center. Results: Some peculiar behaviors were observed such as domestic violence and a better adherence to treatment during their participation in a supportive group with other women. Conclusion: The study pointed out the relevance of trying to understand alcoholism in women with all its peculiarities and the possible impact of this understanding in future interventions.

Key words: woman, alcoholism, sort, violence, feminine drinking.
\end{abstract}

Unidade de Tratamento de Alcoolistas do Instituto Philippe Pinel (UTA/IPP) (Cesar BAL)

Pesquisa realizada na Unidade de Tratamento de Alcoolistas do IPP, Rio de Janeiro

Recebido

18-07-06

Aprovado

26-10-06

Correspondência para: Beatriz Aceti Lenz Cesar

Avenida Venceslau Brás, 65 - Botafogo - 22290-140 - Rio de Janeiro-RJ - Tel.: 2542-3049 - ramais 2045 e 2044 - e-mail: beatrizlenz@yahoo.com.br 


\section{Introdução}

No decorrer dos anos observamos crescente interesse pelo alcoolismo feminino, e a presença ou a ausência de mulheres nos serviços de saúde tem sido o foco central das discussões. Durante muito tempo o alcoolismo esteve identificado com a população masculina, considerando resultados que apontavam para uma prevalência mínima de mulheres nos serviços de saúde especializados. Vanicelli e Nash (1984) revisaram 530 estudos avaliando o tratamento de alcoolismo e constataram que apenas $7 \%$ da população estudada era de mulheres. Hagnell e Tunving (1972), num estudo nos Estados Unidos com uma amostra de 2.612 indivíduos, encontraram apenas uma mulher alcoolista. As taxas de abuso e dependência de álcool chegavam a dois homens para uma mulher nos Estados Unidos, e as taxas nos tratamentos eram de quatro a cinco homens para uma mulher (Blume, 1990). Na América Latina essa proporção variava de 5:1 a 14:1 (Santana e Almeida Filho, 1987). Borini e Silva (1989), Cardim e Azevedo (1995) e Hochgraf et al. (1990) também apontaram o elevado número de alcoolistas homens nos serviços especificamente no Brasil.

A ausência de mulheres nos serviços, evidenciada através desses estudos, abriu um leque de discussões que foram tanto da visão do alcoolismo como um problema predominantemente masculino (Keller, 1980) à tentativa de investigar essa questão compreendendo o comportamento social diante do alcoolismo em mulheres. Robbins (1968) sugeriu que o alcoolismo vinculado ao sexo masculino seria basicamente de natureza social, e que a ausência de mulheres nessa categoria poderia estar vinculada a uma dupla moral implícita na prática do consumo de álcool. Essa dupla moral não só não favoreceria o consumo entre mulheres como aumentaria o estigma para com as mulheres que bebem, impondo ao sexo feminino padrões mais rígidos.

Blume (1986) enfatizou o aumento do número de mulheres com problemas associados ao consumo de álcool a partir da Segunda Guerra, na intenção de problematizar as discussões focando o processo histórico e o contexto sociocultural, que poderiam ter influência sobre a questão. No bojo dessas reflexões, autores como Robbins e Martin (1993), Madrigal (1993), Hochgraf (1995), Oliveira e Silva (2002), apontaram a importância de investigar as especificidades do alcoolismo feminino considerando questões pertinentes à mulher no espaço social. Seus achados sugerem a importância de levar em conta as diferenças de gênero no alcoolismo, refletidas no comportamento de beber de homens e mulheres, o que implica poder ponderar algumas afirmações em torno do alcoolismo em mulheres, como no caso da invisibilidade nos serviços, e apontar para o quanto as peculiaridades do alcoolismo feminino podem interferir nas ações de saúde que envolvem essa problemática, tornando-as mais efetivas.

Diante do exposto, o presente estudo tem como objetivo discutir as peculiaridades do alcoolismo feminino e a importância do seu significado nos serviços especializados.

\section{Métodos}

\section{Local da pesquisa}

A pesquisa foi realizada na Unidade de Tratamento de Alcoolistas do Instituto Philippe Pinel (UTA/IPP) no Rio de
Janeiro. Criada em 1985, a unidade é um serviço especializado que visa ao tratamento e à prevenção dos problemas decorrentes do uso abusivo de bebidas alcoólicas, com objetivos de assistência, ensino e pesquisa. A unidade possui 20 leitos para internação, sendo 15 para homens e cinco para mulheres. Prioriza a ambulatorização e trabalha com o enfoque da redução de danos. A triagem inicial é feita na recepção, com os encaminhamentos necessários para a UTA. Na unidade é realizada uma segunda triagem para início de tratamento. O procedimento do serviço pode incluir ou não a internação. A indicação pode ser desintoxicação ambulatorial e/ou encaminhamento direto para tratamento ambulatorial.

No ambulatório há psicoterapia individual, em grupo, terapia medicamentosa, grupos de família, grupos operativos, hospital-dia e o grupo de mulheres. Criado em 2001, o grupo de mulheres tem como objetivo mudanças nas ações de saúde que envolvam o tratamento de mulheres alcoolistas.

\section{Procedimentos}

Os dados desse estudo são decorrentes de um recorte de pesquisa sobre 0 alcoolismo feminino. 0 estudo foi realizado com nove integrantes do grupo de mulheres da instituição, e o critério de inclusão era participar dele há pelo menos um ano. O propósito desse critério era proporcionar reflexão e discussão em torno das mulheres que já estavam em tratamento, especialmente num espaço direcionado às mulheres. As entrevistadas tinham idades entre 40 e 52 anos e perfil socioeconômico diferenciado.

Foram feitas entrevistas individuais semi-estruturadas. A intenção era ter um roteiro prévio, porém dando espaço de liberdade para as entrevistadas fazerem associações concernentes à sua história de vida que considerassem relevantes. Dessa forma, as afirmações poderiam trazer elementos significativos para a investigação, principalmente se esses elementos fossem coincidentes. Buscaram-se, dentro dos objetivos desse método, a descrição do caso individual, a compreensão das especificidades culturais mais profundas dos grupos, a comparabilidade de diversos casos (Minayo, 1992).

As entrevistas foram gravadas e posteriormente transcritas. Foram observadas e garantidas todas as questões éticas de acordo com a Resolução 196/96. O protocolo de pesquisa foi aprovado pelo Comitê de Ética em Pesquisa da instituição e todos os pacientes assinaram um termo de consentimento livre e esclarecido antes que qualquer procedimento do estudo fosse aplicado.

\section{Resultados}

Com relação ao local onde bebem, 90\% declararam beber no âmbito da esfera privada e diferenciaram esse comportamento do beber na esfera pública. Setenta por cento afirmam ter sofrido algum tipo de violência física/sexual na infância e/ou adolescência por parte de parente próximo. Desses $70 \%$, duas começaram a beber na infância; três, na adolescência e uma, na idade adulta. No momento da entrevista, seis das nove mulheres entrevistadas eram ou foram casadas, sendo que cinco delas tiveram parceiros (maridos 
ou não) alcoolistas. Oitenta por cento das casadas sofreram violências doméstica e sexual por parte dos companheiros e todas haviam sofrido algum tipo de violência sexual/física na infância ou na adolescência. A possibilidade de ter controle sobre o beber foi trazida por $40 \%$. Entre as situações exemplificadas sobre esse controle, o período de gravidez e amamentação dos filhos foi o ponto comum. Verificou-se maior adesão ao tratamento após a criação de um espaço de tratamento diferenciado para mulheres.

\section{Discussão}

A percentagem alta de mulheres que bebem privadamente não atesta que mulheres alcoolistas não bebam em público. Porém a representatividade dada por elas ao beber na esfera privada, como o comportamento que revela o seu alcoolismo, parece apontar uma particularidade do beber feminino.

Estudos comparativos de gênero descrevem comportamentos diferenciados para homens e mulheres alcoolistas. As expressões desses comportamentos são originadas na formação, na educação de meninos e meninas, quando a identidade de gênero vai se constituindo (Robbins e Martin, 1993). Achados desses autores apontam estilos de desvios de gênero em que o comportamento dos homens alcoolizados seria um com reações para fora, externalizado, enquanto as mulheres tenderiam a um comportamento mais retraído, ficando menos expostas e internalizando emoções.

Outro aspecto a considerar seria a preservação da auto-imagem, relacionada ao preconceito diante das mulheres que bebem: cobrança diante de papéis a cumprir, como no caso da maternidade, ou posturas não consideradas femininas socialmente. A repressão ao consumo de álcool pelas mulheres provoca sentimentos de culpa (Smart, 1980), podendo ter como conseqüência um beber escondido. Ao mesmo tempo, essa autopreservação aponta também para um autocuidado, que parece ter relação com a possibilidade de controle sobre o beber. Todavia achados de outro estudo verificaram, num período de seis meses de tratamento, taxa de abstinência menor entre as mulheres do que entre os homens (Hochgraf, 1995). Ao mesmo tempo foi observado que era mais fácil para as mulheres reduzir o consumo do que manter a abstinência, indicando, sob certo ângulo, que elas teriam mais controle sobre o beber do que os homens, mantendo-se bebedoras sociais com mais facilidade do que eles (Hochgraf, 1995).

A abstinência observada no período de gravidez e amamentação dos filhos na nossa amostra sugere que aspectos relacionados à diferença de gênero no alcoolismo podem afetar o comportamento de beber. A função social da mulher enquanto cuidadora poderia funcionar como freio para 0 ato de beber (Robbins e Martin, 1993). Dados de maiores recaídas em mulheres que têm menos filhos morando com elas foram encontrados por Hochgraf (1995).

A alta taxa de mulheres vítimas de violência física/ sexual na infância/adolescência, coincidindo com o fato de começarem a beber naquela ocasião, foi dado relevante da pesquisa e aponta a perspectiva de que a violência pode contribuir para gerar alcoolismo. 0 mesmo já foi descrito por
Windle et al. (1995), cujos achados indicaram que mulheres alcoolistas têm quatro vezes mais história de abuso sexual na infância do que homens, e por estudos recentes de Simpson e Miller (2002), que apontam índices mais altos de abuso físico/sexual na infância em mulheres alcoolistas ou que fazem uso de substâncias psicoativas do que em mulheres que não o fazem.

As casadas vítimas de abuso na infância/adolescência também sofreram violência doméstica por parte dos companheiros. Alguns estudos relatam achados com índices elevados de mulheres alcoolistas vítimas de violência doméstica (Miller et al., 2000). Entretanto não foi detectada relação do abuso sofrido na infância com a perpetuação da violência doméstica sofrida na vida adulta. Sugere-se que essa seja questão para futuras pesquisas, devido à sua relevância e à sua complexidade.

A questão do abuso físico e sexual na infância é extremamente delicada. A naturalidade da ambivalência afetiva no processo de desenvolvimento na infância e na adolescência aponta que em situações de abuso por pessoas da família, quando a criança fica dividida entre o amor e o ódio diante da violência física e emocional, a ambivalência assume proporções graves que a criança não tem condições de suportar (Azevedo, 2001). A situação se agrava devido à baixa notificação e pela dificuldade em lidar com a questão por ser 0 abusador alguém da família, acarretando medo e sentimentos de culpa (Pizá, 1999). Ainda há a dificuldade, encontrada pelos profissionais de saúde, de se sentir à vontade para lidar com essa questão, incluindo a ausência de perguntas dessa natureza nas anamneses realizadas nos serviços (Zilberman e Blume, 2005).

A porcentagem de mulheres que sofreram algum tipo de violência na infância/adolescência casadas com companheiros alcoolistas encontra-se de acordo com os estudos de Gomberg (1981), cujos achados indicam que mulheres vítimas de abuso físico ou sexual tendem a se casar com homens alcoolistas. Entretanto seus depoimentos apontam para a impossibilidade de construir relações mais duradouras com companheiros que não tenham problemas de alcoolismo. A maioria delas teve contato com a bebida muito cedo e conviveu com amigos e amigas também alcoolistas, o que propiciava a relação com uma pessoa que bebesse.

A criação do espaço de tratamento específico para mulheres - caracterizado pelo Grupo de Mulheres Alcoolistas - no serviço onde foi realizada a pesquisa revelou adesão ao tratamento de $70 \%$ das mulheres atendidas contra evasão de $90 \%$ antes da criação do espaço. Esses dados, no entanto, não são suficientes para indicar a necessidade de tratamentos separados para homens e mulheres, mas sugerem que mudanças nos modelos de atenção junto às mulheres alcoolistas poderiam favorecer ações mais efetivas por parte das equipes de saúde nessa área.

Não é incomum mulheres procurarem os serviços de saúde com queixas vagas, encobrindo o real problema que as afeta. Mulheres alcoolistas tendem a procurar serviços de clínica ginecológica ou médica referindo-se a sintomas físicos ginecológicos ou a sintomas psíquicos, como ansiedade ou depressão, sem, contudo, revelar seu problema de alcoolismo. 
Situação semelhante é encontrada por mulheres vítimas de violência (Zilberman e Blume, 2005).

\section{Conclusão}

O fato de o estudo ter uma população pequena de mulheres lhe confere algumas limitações quanto à afirmação de resultados. No entanto os resultados obtidos nesse

\section{Referências}

Azevedo EC. Atendimento psicanalítico a crianças e adolescentes vítimas de abuso sexual. Psicologia Ciência e Profissão, 21(4): 66-77, 2001.

Blume S. Chemical dependency in women: important issues. Am J Drug Alcohol Abuse, 16(3 \& 4): 297-307, 1990.

Blume S. Women and alcohol. JAMA, 256: 1467-70, 1986.

Borini P, Silva CO. Aspectos demográficos, epidemiológicos e sociais do alcoolismo: uma análise de alcoolistas internados em hospital psiquiátrico. Rev ABP APAL, 11(3): 89-96, 1989.

Cardim MS, Azevedo BA. Antecedentes familiares na determinação da gravidade do alcoolismo. Informe Psiq, 14(1): 5-12, 1995.

Gomberg ESL. Women, sex roles, and alcohol problems. Prof Psychol, 12: 146-55, 1981.

Hagnell O, Tunving K. Prevalence and nature of alcoholism in a population. Soc Psychiatry, 7: 190-201, 1972.

Hochgraf PB. Alcoolismo feminino: comparação de características sociodemográficas e padrão de evolução entre homens e mulheres alcoolistas. Tese de doutorado apresentada à USP, 1995.

Hochgraf PB, Tucci MR, Zilberman ML, Andrade AG. Comparação entre mulheres e homens alcoolistas em relação a dados sociodemográficos e outras características relacionadas ao uso de álcool. Rev ABPAPAL, 12(1, 2, 3, 4): 25-30, 1990.

Keller M. Concepções sobre o alcoolismo. Rev ABPAPAL, 2(2): 93-100, 1980.

Madrigal E. Patrones de consumo del alcohol y de sustancias psicoactivas en la mujer. In: Gómez-Gómez E, editor. Género, mujer y salud en las Américas, Washington DC: Organización Panamericana de la Salud; 1993. p. 163-77.

Miller BA, Wilsmack SC, Cunradi CB. Family violence and victimization: recorte da pesquisa apontam a relevância, já sinalizada por outros autores, de estudos que dêem seguimento a pesquisas relacionadas ao alcoolismo feminino e suas peculiaridades. Nesse contexto, a discussão levantada pelos resultados reforça o diálogo sobre a importância de mudanças nas ações de saúde que envolvam o atendimento a mulheres alcoolistas levando-se em conta sua complexidade. treatment issues for women with alcohol problems. Alcohol Clin Exp Res, 24(8): 1287-97, 2000.

Minayo MCS. O desafio do conhecimento: pesquisa qualitativa em saúde. São Paulo: Hucitec/Rio de Janeiro: ABRASCO; 1992.

Oliveira e Silva PC. Alcoolismo feminino: um estudo sob a perspectiva de gênero. Dissertação de mestrado. Programa de Estudos Interdisciplinares de Comunidades e Ecologia Social (EICOS)/UFRJ, 2002.

Pizá GA. Violência silenciosa do incesto. Revista Ciência e Saúde Coletiva, 1999.

Robbins CA, Martin SS. Gender, styles of deviance, and drinking problems. J Health Soc Behav, 34: 302-21, 1993.

Robbins L. Drinking behavior of young urban negro men. Q J Stud Alcohol, 29(3): 657-84, 1968.

Santana VS, Almeida Filho N. Alcoolismo e consumo de álcool: resumo de achados epidemiológicos. Rev ABPAPAL, 9: 15-22, 1987.

Simpson TL, Miller WR. Comcomitance between childhood sexual and physical abuse and substance use problems: a review. Clin Psychol Rev, 22(1): 27-77, 2002.

Smart R. Identificação de alcoólatras e bebedores problemáticos. Rev Bras Psiquiatr, 2(2): 105-13, 1980.

Vannicelli M, Nash L. Effect of sex bias on women's studies on alcoholism. Alcohol Clin Exp Res, 8: 334-6, 1984.

Windle M, Windle RC, Scheidt DM, Miller GB. Physical and sexual abuse and associated mental disorders among alcoholic inpatient. Am J Psychiatry, 152: 1322-8, 1995.

Zilberman ML, Blume SB. Violência doméstica, abuso de álcool e substâncias psicoativas. Rev Bras Psiquiatr, 27(Supl II): S51-5, 2005. 